\title{
PRNP Gene
}

National Cancer Institute

\section{Source}

National Cancer Institute. PRNP Gene. NCI Thesaurus. Code C71442.

This gene is involved in prion diseases. 